\title{
Guidelines for Guidelines: An Assessment of the American Society of Breast Surgeons Contralateral Prophylactic Mastectomy Consensus Statement
}

\author{
Todd M. Tuttle, $\mathrm{MD}^{1}$, Andrea V. Barrio, $\mathrm{MD}^{2}$, V. Suzanne Klimberg, $\mathrm{MD}^{3}$, Armando E. Giuliano, $\mathrm{MD}^{4}$, \\ Mariana Chavez-MacGregor, MD ${ }^{5}$, Heather A. Thompson Buum ${ }^{6}$, and Kelly M. McMasters, MD, PhD ${ }^{7}$ \\ ${ }^{1}$ University of Minnesota, Minneapolis, MN ; ${ }^{2}$ Memorial Sloan Kettering Cancer Center, New York, NY; ${ }^{3}$ University of \\ Arkansas, Little Rock, AR; ${ }^{4}$ Cedars-Sinai, Los Angeles, CA; ${ }^{5}$ Anderson Cancer Center, Houston, TX; ${ }^{6}$ University of \\ Minnesota, Minneapolis, MN; ${ }^{7}$ University of Louisville, Louisville, KY
}

The rates of contralateral prophylactic mastectomy (CPM) have markedly increased in the US over the past 2 decades. ${ }^{1-3}$ These trends have been observed in all patient age groups, cancer stages, races, and in all geographic regions of the US. In the most recently published analyses from the Surveillance Epidemiology and End Results database, the CPM rates were still increasing with no plateau. ${ }^{3}$ Most patients who undergo CPM do not have strong genetic or familial risk factors for developing contralateral breast cancer. ${ }^{4}$ Recent survey studies have demonstrated that breast cancer patients substantially overestimate the risk of contralateral breast cancer and have unrealistic outcomes from CPM. ${ }^{5,6}$ Moreover, in a survey study among active members of the American Society of Breast Surgeons (ASBrS), Yao et al. concluded that $39.2 \%$ of respondents had a 'low level of knowledge about CPM'. ${ }^{7}$ Given the gaps in knowledge among both patients and surgeons, the publication of the ASBrS consensus statement is timely.

In 1993, the Society of Surgical Oncology (SSO) developed a position statement on the use of CPM, which was most recently edited and updated in March 2007. ${ }^{8}$ Since the last revision of this position statement, many important studies have been published evaluating the risks of contralateral breast cancer, outcomes after CPM, and patients' perceptions and preferences. The ASBrS

(C) Society of Surgical Oncology 2016

First Received: 8 September 2016;

Published Online: 25 October 2016

T. M. Tuttle, MD

e-mail: tutt1006@umn.edu consensus statement appropriately incorporates most of this relevant recent research.

In the strongest language to date, the consensus statement recommends that CPM should be 'discouraged' for patients with an average risk of contralateral breast cancer. This population of patients represents the vast majority of women who undergo CPM in the US. The statement further concludes that 'CPM should be considered' for selected groups at significant risk of contralateral breast cancer (including carriers of BRCA 1 or 2 deleterious mutations). Furthermore, 'CPM can be considered' for selected groups at lower risk of contralateral breast cancer (including other gene mutation carriers). Additionally, 'CPM may be considered' for non-oncologic reasons (including limiting contralateral breast surveillance). Finally, 'CPM should be discouraged' for patients with advanced primary-stage breast cancer and patients who are in overall poor health or at very high risk of associated complications.

In recent years, there has been a rapid proliferation in the number and scope of published clinical practice guidelines and consensus statements. To address the substantial variation in the clinical guideline development processes, the Institute of Medicine (IOM) published eight standards in 'Clinical Practice Guidelines We Can Trust' in 2011. ${ }^{9}$ Although there are subtle differences between consensus statements and clinical practice guidelines, both should be transparent, multidisciplinary, evidence-based, and intended to provide guidance to clinicians and patients. The ASBrS CPM consensus statement adheres to some, but not all, of the IOM standards. For example, the IOM recommends that the clinical guideline development group be multidisciplinary, balanced, and include current/former 
patients and patient advocates. Although the author list of the ASBrS consensus statement is primarily breast surgeons, plastic surgeons and a patient advocate/survivor are also included. Ideally, medical oncologists, breast radiologists, and other members of the multidisciplinary breast cancer team should have been represented in this particular guideline. Moreover, given the impact of patient preference and substantial patient misconceptions regarding contralateral breast cancer and CPM, participation of a clinical psychologist would have been contributory.

The IOM also recommends performance of a systematic review of existing data to support clinical practice guideline development. The American Society of Clinical Oncology (ASCO) also endorses 'comprehensive systematic reviews of the literature to support new guideline recommendations'. ${ }^{10}$ ASCO has outlined a series of necessary steps to plan and conduct a systematic review for a clinical guideline. The 'literature review' performed by the ASBrS consensus statement group does not meet these standards. It did not indicate how the literature review was conducted and did not detail the criteria for including and excluding studies from evidence review. Moreover, a metaanalysis of data derived from a systemic review was not presented in the consensus statement. In addition, the final conclusions lack a rating of the strength of the recommendations and also level of confidence in the evidence underpinning them.

Finally, the IOM recommends that external reviewers should include a full spectrum of relevant stakeholders. For a CPM guideline, other relevant stakeholders would include the SSO, ASCO, American Society of Plastic Surgeons, and American College of Radiology. The impact of a CPM clinical practice guideline would be greatly enhanced by inclusion of other relevant societies in addition to the ASBrS. By comparison, the 'Consensus Guideline on Margins for Breast-Conserving Surgery' included a systemic review and a meta-analysis and was endorsed by the SSO, American Society for Radiation Oncology, ASBrS, and ASCO. ${ }^{11}$

Despite our criticisms of the guideline development process, the authors of this editorial generally agree with the conclusions from the ASBrS consensus statement. We acknowledge that the development of an evidenced-based consensus guideline on CPM is a complex and unique process. In addition to traditional outcome measurements (risk of contralateral breast cancer, survival rates, and complications), the guideline must also consider patient preferences (breast symmetry, avoidance of future surveillance, and anxiety). Although the recommendations presented in the consensus statement were not supported by a rigorous systematic review/meta-analysis, the conclusions from the ASBrS consensus statement are practical and apply to specific populations of breast cancer patients.

The primary aim of a clinical practice guideline or consensus statement is to assist physician and patient healthcare decisions for specific circumstances. Evidencebased cancer guidelines can 'move the needle' and substantially impact national patterns of care. We hope that the much needed recommendations from the ASBrS consensus statement will reverse the trends of increasing rates of CPM, a procedure that increases complications and provides no meaningful oncologic benefit for most breast cancer patients.

\section{REFERENCES}

1. Tuttle TM, Habermann EB, Grund EH, et al. Increasing use of contralateral prophylactic mastectomy for breast cancer patients: a trend toward more aggressive surgical treatment. J Clin Oncol. 2007;25:5203-09.

2. Yao K, Stewart AK, Winchester DJ, Winchester DP. Trends in contralateral prophylactic mastectomy for unilateral cancer: a report from the National Cancer Data Base, 1998-2007. Ann Surg Oncol. 2010;17(10):2554-62.

3. Wong SM, Freedman RA, Sagara Y, Aydogan F, Barry WT, Golshan M. Growing use of contralateral prophylactic mastectomy despite no improvement in long-term survival for invasive breast cancer. Ann Surg. 2016. doi:10.1097/SLA.0000000000001698.

4. Hawley ST, Jagsi R, Morrow M, Janz NK, Hamilton A, Graff JJ, et al. Social and clinical determinants of contralateral prophylactic mastectomy. JAMA Surg. 2014;149(6):582-9.

5. Abbott A, Rueth N, Pappas-Varco S, et al. Perceptions of contralateral breast cancer: an overestimation of risk. Ann Surg Oncol. 2011;18(11):3129-36.

6. Rosenberg SM, Tracy MS, Meyer ME, et al. Perceptions, knowledge, and satisfaction with contralateral prophylactic mastectomy among young women with breast cancer: a crosssectional survey. Ann Intern Med. 2013;159(6):373-81.

7. Yao K, Belkora J, Sisco M, et al. Survey of the deficits in surgeons' knowledge of contralateral prophylactic mastectomy. JAMA Surg. 2016;151(4):391-3.

8. Giuliano AE, Boolbol S, Degnim A, Kuerer H, Leitch AM, Morrow M. Society of Surgical Oncology: position statement on prophylactic mastectomy. Approved by the Society of Surgical Oncology Executive Council, March 2007. Ann Surg Oncol. 2007;14(9):2425-7.

9. Graham R, Mancher M, Wolman DM, Greenfield S, Steinberg E (eds). Clinical practice guidelines we can trust: Committee on Standards for Developing Trustworthy Clinical Practice Guidelines. Washington, DC: National Academies Press; 2011.

10. Somerfield MR, Einhaus K, Hagerty KL, Brouwers MC, Seidenfeld J, Lyman GH; American Society of Clinical Oncology. American Society of Clinical Oncology clinical practice guidelines: opportunities and challenges. $J$ Clin Oncol. 2008;26(24):4022-6.

11. Moran MS, Schnitt SJ, Giuliano AE, Harris JR, Khan SA, Horton $\mathrm{J}$, et al. Society of Surgical Oncology-American Society for Radiation Oncology consensus guideline on margins for breastconserving surgery with whole-breast irradiation in stages I and II invasive breast cancer. Ann Surg Oncol. 2014;21(3):704-16. 